\title{
A INFLUÊNCIA DO CONSUMO DE BEBIDAS ALCOÓLICAS NA OCORRÊNCIA DE VIOLÊNCIA POR PARCEIRO ÍNTIMO: REVISÃO INTEGRATIVA
}

\author{
Welton Souza Campos de Araújo ${ }^{1}$ \\ Andrey Ferreira da Silva ${ }^{2}$ \\ Fernanda Matheus Estrela ${ }^{3}$ \\ Josinete Gonçalves dos Santos Lírio ${ }^{4}$ \\ Moniky Araújo da Cruz \\ Jemima Raquel Lopes Santos ${ }^{6}$ \\ Álvaro Pereira ${ }^{7}$
}

\begin{abstract}
ARAÚJO, W. S. C. de; SILVA, A. F. da; ESTRELA, F. M.; LÍRIO, J. G. dos S.; CRUZ, M. A. da; SANTOS, J. R. L.; PEREIRA, A. A influência do consumo de bebidas alcoólicas na ocorrência de violência por parceiro íntimo: revisão integrativa. Arq. Cienc. Saúde UNIPAR, Umuarama, v. 22, n. 2, p. 117-122, maio/ago. 2018.
\end{abstract}

\begin{abstract}
RESUMO: A violência por parceiro íntimo, muitas vezes, tem o uso do álcool e seus efeitos colaterais como elementos precipitadores e intensificadores das condutas violentas. Diante desse pressuposto, tem-se por objetivo identificar na literatura científica, nacional e internacional, as evidências científicas acerca da influência do consumo de bebidas alcoólicas na ocorrência de violência por parceiro íntimo. Trata-se de uma revisão integrativa da literatura realizada nas bases de dados Medical Literature Analysis and Retrieval System Online, Scientific Electronic Library Online, Literatura Latino-Americana e do Caribe em Ciências da Saúde e Base de dados de enfermagem, nas línguas portuguesa e inglesa, não havendo artigos em espanhol, com os descritores: consumo de bebidas alcoólicas, violência contra a mulher e violência conjugal. Considerou-se como critérios de inclusão: artigos originais, publicados no período de 2011 a 2016, disponíveis gratuitamente nos idiomas português, inglês ou espanhol, excluindo assim artigos fora dos critérios de inclusão, além de teses, dissertações, monografias, livros, capítulos de livros, documentos governamentais e seminários. Foram encontrados 56 artigos, contudo, somente 7 atenderam aos critérios de inclusão e exclusão e responderam a questão de revisão. Dentre os selecionados, realizou-se uma análise, a qual correspondeu à síntese das evidências disponíveis nos artigos que compõem o corpus do estudo. A literatura nacional e internacional desponta para o consumo de bebidas alcoólicas dentro da relação conjugal, como um elemento que contribui de forma significativa para uma evidente propensão à prática da violência por parceiro íntimo, pois está relacionado com a instabilidade emocional e a reação contra as agressões perpetradas pelo parceiro íntimo.
\end{abstract}

PALAVRAS-CHAVE: Consumo de bebidas alcoólicas. Violência contra a mulher. Violência por parceiro íntimo. Saúde da mulher.

\section{THE INFLUENCE OF CONSUMPTION OF ALCOHOLIC BEVERAGES IN THE OCCURRENCE OF INTIMATE PARTNER VIOLENCE: A COMPREHENSIVE REVIEW}

\begin{abstract}
Intimate partner violence often has the consumption of alcohol and its side effects as precipitating and intensifying elements of violent behavior. Based on this assumption, the purpose of this study is to identify scientific evidence in the national and international scientific literature on the influence of alcohol consumption on the occurrence of violence by an intimate partner. This is an integrative literature review using the Medical Literature Analysis and Retrieval System Online databases, the Scientific Electronic Library Online, the Latin American and Caribbean Literature on Health Sciences and the Nursing Database, in both Portuguese and English, since there were no papers published in Spanish with the descriptors: alcohol consumption, violence against women and marital violence. Inclusion criteria were: novelty articles, published between 2011 and 2016, available free of charge in Portuguese, English or Spanish, thus excluding any articles outside the inclusion criteria, as well as theses, dissertations, monographs, books, book chapters, government documents and seminars. A total of 56 articles were found. However, only seven met the inclusion criteria and answered the review question. Among those selected, an analysis was performed, which corresponded to the synthesis of the available evidence in the articles that make up the study corpus. The national and international literature emerges for the consumption of alcoholic beverages within the marital relationship as an element that significantly contributes to an evident propensity to practice intimate partner violence, since it is related to emotional instability and reaction against aggressions perpetrated by the intimate partner.
\end{abstract}

KEYWORDS: Consumption of alcoholic beverages. Intimate partner violence. Violence against women. Women's health.

DOI: $10.25110 /$ arqsaude.v22i2.2018.6380

${ }^{1}$ Graduando de Enfermagem da Universidade Federal da Bahia. Endereço: Travessa Sauípe, nº 59, quadra 04, STIEP, Salvador, Ba. Cep. 41770-610. E-mail: weltonaraujo@gmail.com

${ }^{2}$ Enfermeiro, Doutorando do Programa de Pós-Graduação em Enfermagem da Universidade Federal da Bahia. Endereço: Rua Caetano Moura, No 130, Apto. 104. Federação, Salvador, Ba. Cep. 40210-341. Tel. E-mail: silva.andrey1991@hotmail.com

${ }^{3}$ Enfermeira, Doutoranda do Programa de Pós-Graduação em Enfermagem da Universidade Federal da Bahia. Endereço: Rua Antônio Moniz, № 04. Bonfim, Salvador, Ba. Cep: 404151-45. E-mail: nanmatheus@yahoo.com.br

${ }^{4}$ Enfermeira, Mestranda do Programa de Pós-Graduação em Enfermagem da Universidade Federal da Bahia. Endereço: Rua José Bonifácio, № 66 . Vila Ruy Barbosa, Salvador, Ba. Cep: 404030-160. Tel. E-mail: josilirio@hotmail.com

${ }^{5}$ Graduanda de Enfermagem da Universidade Federal da Bahia. Endereço: Ladeira quinta dos lázaros, № 78, Apto 203. Baixa de Quintas, Salvador, Ba. Cep. 40300-415. E-mail: monikyac@hotmail.com

${ }^{6}$ Graduanda do Bacharelado Interdisciplinar de Saúde da Universidade Federal da Bahia. Endereço: Rua da Independência, No 79. CIA 1, Simões Filho, Ba. Cep.49700-000. E-mail: jemima.raquel03@outlook.com

${ }^{7}$ Enfermeiro, Doutor em Filosofia da Enfermagem, Professor Títular da Escola de Enfermagem da Universidade Federal da Bahia. Endereço: Rua Santa Isabella, 100/1704 - B. Engenho Velho da Federação, Salvador, Ba. Cep: 40221-225 Tel. E-mail: alvaro_pereira_ba@yahoo.com.br 


\section{Introdução}

A violência por parceiro íntimo (VPI) traduz-se como qualquer ação ou omissão contra a pessoa com a qual se tem uma relação íntima, tendo muitas vezes o uso das bebidas alcoólicas e seus efeitos colaterais como elementos precipitadores e intensificadores das condutas violentas. No âmbito conjugal, a violência representa uma relação de assimetria alicerçada na desigualdade existente entre os gêneros (SOUSA et al., 2016).

Posto isto, quando homens e mulheres desempenham seus papéis sociais de forma dissemelhante, muitas vezes manifestados em formato deletério no interior das relações conjugais, reforçar-se o abismo social existente entre os gêneros. Neste trilho, a violência por parceiro íntimo se caracteriza pelo padrão de comportamento abusivo e coercitivo de um indivíduo contra sua/seu parceira(o) que pode incluir dano físico, psicológico, sexual, isolamento progressivo, perseguição, privação, intimidação e/ou coerção repetitiva. Neste sentido, Vieira, Perdona e Santos (2011) revelam que na Argentina, Chile e EUA a prevalência e variabilidade de violência por parceiro íntimo têm alcançado números entre $15-71 \%$. Ainda no cenário internacional, estudo norte americano afirmou que as mulheres são as principais vítimas desse tipo de violência (LINDNER et al., 2015).

Diversos fatores são contribuintes para a prática da VPI, dentre eles, destaca-se o uso de bebidas alcoólicas. Pesquisa realizada em municípios brasileiros, com homens e mulheres em situação de violência por parceiro íntimo, revelou que para cada 10 homens em situação deste tipo de violência, quatro afirmaram ter ingerido bebida alcoólica antes, durante e depois da prática da agressiva. Além disso, o mesmo estudo denuncia o aumento da violência, ao apontar para a física como a principal forma manifestada (LINDNER et al., 2015; ZALESKI et al., 2010), ratificando pesquisa realizada com 50 casais, na Unidade Integral de Avaliação de Violência de Gênero em Sevilha, Espanha, em 2013 e 2014, revelou a existência de um aumento significativo da prática de VPI, motivada pela ingestão de bebidas alcoólicas (GIMÉNEZ; GARCÍA; MANCHA, 2014).

Diante do aumento da prevalência de VPI, tendo a mulher como principal vítima, a Lei 11.340/06 foi promulgada no Brasil com o intuito de coibir a prática desse tipo de violência. Denominada Maria da Penha, tal lei versa sobre medidas punitivas e espaços de educação para agressores, além da criação de Varas especializadas para o atendimento à mulher em situação de violência (BRASIL, 2006). No entanto, percebe-se a necessidade de desenvolver estudos que tratem do consumo de bebidas alcoólicas como elemento precipitador e intensificador da violência por parceiro íntimo, de modo a ofertar subsídios para o desenvolvimento e reformulação de políticas públicas para o seu enfrentamento. Ante essa necessidade, levantou-se a seguinte questão de revisão: quais as evidências científicas na literatura científica nacional e internacional acerca da influência do consumo de bebidas alcoólicas na ocorrência de violência por parceiro íntimo? Sendo assim, este estudo tem como objetivo identificar na literatura científica nacional e internacional as evidências científicas acerca da influência do consumo de bebidas alcoólicas na ocorrência de violência por parceiro íntimo.

\section{Metodologia}

Trata-se de um estudo de revisão integrativa da literatura que para Mendes, Silveira e Galvão (2008) se propõe a sintetizar as evidências disponíveis na literatura científica sobre determinado fenômeno, aprofundando o conhecimento relacionado ao assunto.

Para melhor andamento desta pesquisa, foram adotadas as etapas preconizadas por Mendes, Silveira e Galvão (2008) que são: identificação do tema e seleção da hipótese, definição dos critérios de inclusão e exclusão de estudos, categorização dos estudos, avaliação dos estudos utilizados na amostragem, interpretação dos resultados, síntese do conhecimento.

Diante da vastidão da temática a ser explorada, foi elencada a seguinte questão de revisão: quais as evidências científicas na literatura científica nacional e internacional acerca da influência do consumo de bebidas alcoólicas na ocorrência de violência por parceiro íntimo?

Para melhor delimitação da busca, utilizou-se os seguintes critérios de inclusão: artigos originais, publicados no período de janeiro de 2011 a dezembro de 2016, disponíveis gratuitamente, online, nos idiomas português, inglês ou espanhol, como critérios de exclusão elencou-se: artigos fora do período pré-estabelecido, revisões de literatura, teses, dissertações, monografias, livros, capítulos de livros, documentos governamentais, artigos não disponíveis gratuitamente na íntegra online. Vale ressaltar que o recorte temporal baseou-se na busca de artigos mais atuais, além disso, artigos duplicados foram considerados uma única vez.

A busca da literatura científica ocorreu nas seguintes bases de dados: Literatura Latino-Americana e do Caribe em Ciências da Saúde (LILACS), Scientific Electronic Library Online (SciELO), Medical Literature Analysis and Retrieval System Online (MEDLINE) e Base de dados de enfermagem (BDENF), por meio da utilização dos seguintes Descritores em Ciências da Saúde (DeCS): "consumo de bebidas alcoólicas", "violência contra a mulher", "violência conjugal", empregando o operador booleano "and" entre as expressões, haja vista que esse operador realiza uma intercessão dos descritores, delimitando ainda mais a busca.

Foram encontrados 56 artigos, sendo 45 na MEDLINE, 8 no LILACS, 3 na BDENF. Após aplicação dos critérios de exclusão e leitura flutuante dos títulos e dos resumos, foram excluídos um total de 49 estudos, sendo 37 por estarem fora do período estudado, 1 por não estar completo, 8 por não contemplarem o objetivo, 1 por ser comentário, 1 por estar duplicado e 1 por não ser artigo original, como mostra o fluxograma a seguir. 
Figura 1: Fluxograma da seleção dos estudos

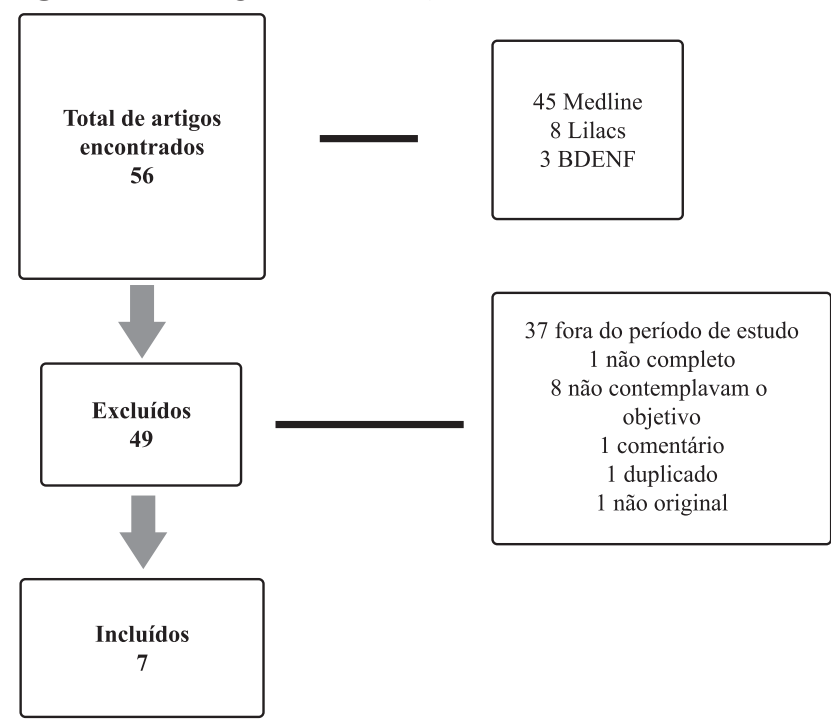

A análise dos dados foi realizada a partir da síntese das evidências disponíveis nos artigos mediante a leitura flutuante, regra de exaustividade, exploração do material, cate- gorização e tratamento dos dados.

Conforme a Lei de Direitos Autorais, Lei n ${ }^{\circ} 12.853$ de 14 de agosto de 2013, que entrou em vigor alterando a Lei $\mathrm{n}^{\circ} 9.610 / 1998$, o estudo atenderá aos aspectos éticos, uma vez que serão respeitados os direitos autorais das pesquisas coletadas (BRASIL, 2013). Ainda, pelo seu perfil científico, a revisão integrativa dispensa a submissão do estudo a um Comitê de Ética em Pesquisa.

\section{Resultados}

Após a aplicação de todos os critérios de exclusão foram selecionados sete artigos para análise. O idioma de prevalência dos artigos foi o inglês (75\%), a principal população estudada nos artigos foram mulheres em situação de violência por parceiro íntimo $(87,5 \%)$, sendo os estabelecimentos de saúde os principais cenários dos estudos (62,5\%). No tocante ao delineamento metodológico utilizado nas pesquisas, houve predomínio do método quantitativo (75\%). O quadro a seguir apresenta a relação de artigos selecionados de acordo com periódico, ano de publicação, país de origem do artigo, autoria, título e objetivos.

Quadro 1: Síntese dos estudos incluídos na revisão integrativa de acordo com o periódico, ano de publicação, país de origem, autoria, títulos e objetivos.

\begin{tabular}{|c|c|c|c|c|c|}
\hline Cód. & Periódico & $\begin{array}{c}\text { Ano de } \\
\text { publicação/ } \\
\text { País de origem }\end{array}$ & Autoria & Título & Objetivo \\
\hline AA1 & $\begin{array}{c}\text { Revista } \\
\text { Baiana de } \\
\text { Enfermagem }\end{array}$ & 2015/Brasil & $\begin{array}{l}\text { SENA, C. D. de; DINIZ, } \\
\text { N. M. F.; CARVALHO, } \\
\text { M. R da S.; OLIVEIRA, } \\
\text { D. S; OLIVEIRA, J. F.; } \\
\text { CAMPOS, M. da L. A. } \\
\text { O. }\end{array}$ & $\begin{array}{l}\text { Ocorrência de violência } \\
\text { doméstica em mulheres } \\
\text { que tiveram parto } \\
\text { prematuro. }\end{array}$ & $\begin{array}{l}\text { Identificar a ocorrência } \\
\text { de violência doméstica } \\
\text { em mulheres que tiveram } \\
\text { parto prematuro }\end{array}$ \\
\hline AA2 & Sage Journals & 2015/Nigéria & $\begin{array}{l}\text { OLOWOOKERE S. } \\
\text { A.; FAWOLE, O. I.; } \\
\text { ADEKANLE D. A.; } \\
\text { ADELEKE N. A.; } \\
\text { ABIOYE-KUTEYI1 E. } \\
\text { A. }\end{array}$ & $\begin{array}{l}\text { Padrões e correlações } \\
\text { da violência do parceiro } \\
\text { íntimo para mulheres } \\
\text { que vivem com HIV/ } \\
\text { AIDS em Osogbo, } \\
\text { sudoeste da Nigéria }\end{array}$ & $\begin{array}{l}\text { Fornecer informações } \\
\text { sobre a relação entre } \\
\text { VPI e HIV/AIDS em } \\
\text { Mulheres portadoras de } \\
\text { HIV/AIDS }\end{array}$ \\
\hline A3 & $\begin{array}{l}\text { Pan Americal } \\
\text { Journal of } \\
\text { Public Health }\end{array}$ & 2015/Brasil & $\begin{array}{l}\text { BARBOSA, J. A. G.; } \\
\text { SOUZA M. C. M. R. de; } \\
\text { FREITAS, M. I. de F. }\end{array}$ & $\begin{array}{l}\text { Violência sexual: } \\
\text { narrativas de mulheres } \\
\text { com transtornos mentais } \\
\text { no Brasil }\end{array}$ & $\begin{array}{l}\text { Compreender o impacto } \\
\text { da violência sexual } \\
\text { sofrida por mulheres } \\
\text { com transtornos mentais } \\
\text { a partir de autorrelato de } \\
\text { suas experiências. }\end{array}$ \\
\hline AA4 & $\begin{array}{l}\text { Indian } \\
\text { Journal of } \\
\text { Medical } \\
\text { Research }\end{array}$ & 2015/Índia & $\begin{array}{l}\text { SHAHINA BEGUM, } \\
\text { BALAIAH DONTA, } \\
\text { SARITHA NAIR, C.P. } \\
\text { PRAKASAM }\end{array}$ & $\begin{array}{l}\text { Fatores sócio- } \\
\text { demográficos associados } \\
\text { à violência doméstica } \\
\text { em favelas urbanas, } \\
\text { Mumbai, Maharashtra, } \\
\text { Índia }\end{array}$ & $\begin{array}{l}\text { Conhecer a prevalência } \\
\text { da violência doméstica e } \\
\text { compreender os fatores } \\
\text { sociodemográficos } \\
\text { associados a ela em } \\
\text { favelas urbanas de } \\
\text { Mumbai, Maharashtra, } \\
\text { Índia. }\end{array}$ \\
\hline
\end{tabular}




\begin{tabular}{|c|l|l|l|l|l|}
\hline AA5 & $\begin{array}{c}\text { Reproductive } \\
\text { Health }\end{array}$ & 2013/Etiópia & $\begin{array}{l}\text { Violência doméstica e } \\
\text { seus preditores entre } \\
\text { Mulheres casadas em } \\
\text { idade reprodutiva em } \\
\text { Fagitalekoma Woreda, } \\
\text { zona Awi, Amara, Oeste } \\
\text { do Noroeste da Etiópia. }\end{array}$ & $\begin{array}{l}\text { Determinar a Magnitude } \\
\text { da violência doméstica } \\
\text { e identificar os seus } \\
\text { preditores entre } \\
\text { mulheres casadas na } \\
\text { idade reprodutiva no } \\
\text { Oeste do Noroeste da } \\
\text { Etiópia. }\end{array}$ \\
\hline ABDULAHI M.
\end{tabular}

A partir da análise feita nos artigos selecionados, foi possível evidenciar as principais influências do consumo de bebidas alcoólicas na prática da Violência por Parceiro Íntimo. Estas se apresentaram como propensão à violência e instabilidade emocional.

\section{Discussão}

Segundo a literatura selecionada para este estudo, o consumo de bebidas alcoólicas e a violência sempre estiveram correlacionados, sendo esta combinação percebida desde tempos remotos. De acordo com os artigos levantados, existe uma possível relação entre ingestão de bebidas alcoólicas e o crescimento dos índices de violência.

No estudo de Semahegn, Belachew e Abdulahi (2013), o consumo de bebidas alcoólicas é apontado como elemento precipitador da violência. Essa ingestão pode estar associada a diversos fatores, como por exemplo, em resposta ao desemprego e/ou dificuldade econômica (OLOWOOKERE et al., 2015). Posto isto, os homens podem usar, de forma abusiva, as bebidas alcoólicas e praticar a violência no âmbito das relações sociais, sendo a parceira íntima a principal vítima. Segundo Eaton et al. (2012), quanto maior a ingestão de bebidas alcoólicas, maiores são as chances de um indivíduo sofrer ou cometer atos violentos, contudo, diante da complexidade desta relação, não se pode restringir tal tendência apenas ao consumo de bebidas alcoólicas (SHAHINA, et al. 2015).

Nessa perspectiva, faz-se necessário a consideração de outros fatores que, associados ao consumo de bebidas alcoólicas, contribuem para a ocorrência de agressões por par- ceiros íntimos, sendo essas: o contexto no qual as bebidas alcoólicas foram consumidas, os valores sociais, as atitudes e expectativas dos consumidores, assim como os efeitos da bebida alcoólica na percepção, coordenação motora, emoção e cognição (SHAHINA et al., 2015).

Observou-se na literatura estudada que a ingestão de bebidas alcoólicas, nas relações conjugais, gera uma propensão para desestabilizar o equilíbrio emocional dos cônjuges, aumentando assim, as chances para a ocorrência dos episódios de violência. Estudo realizado na Índia evidenciou que o consumo de bebidas alcóolicas possui associação com a perpetração da violência, gerando repercussões para as mulheres, a exemplo da depressão (ANINDITA et al., 2013).

Para aquele que está sobre efeito do álcool, há repercussões relacionadas ao desequilíbrio fisiológico, o qual pode ser percebido em diversas atitudes que modificam o comportamento do indivíduo. O primeiro comportamento percebido quando se inicia o consumo de bebidas alcoólicas é a desinibição emocional com manifestações de euforia, comunicação excessiva e autoconfiança, (SENA et al., 2015; BARBOSA; SOUZA; FREITAS, 2015; SHAHINA et al., 2015). Tais sinais nos sinalizam para a dificuldade de tomar decisões de forma coerente e de controlar suas ações. Nesse sentido, os estudos levantados revelam que existe vinculação entre o consumo de bebidas alcoólicas e a prática da violência por parceiro íntimo, a partir da sua influência na instabilidade emocional.

À medida que a concentração etílica na corrente sanguínea aumenta, devido a continuidade do consumo de bebidas alcoólicas, o consumidor entra no estágio de excitação, o que segundo Senna et al. (2015) eleva a instabilidade 
emocional, prejudica sua percepção, memória, compreensão, diminuição da resposta sensitiva e retardo da resposta reativa. Intensificando-se ainda mais a ingesta de bebidas alcoólicas, ocorrerão manifestações comportamentais mais exacerbadas, acompanhadas ou não de desorientação, confusão mental, adormecimento, prejuízo na visão, mobilidade e dimensionamento de espaço, além disso, o indivíduo apresenta fala arrastada, apatia e letargia. Segundo os artigos levantados, estas manifestações comportamentais e emocionais, expressas num ambiente conjugal hostil, podem resultar em violência. VPI (SENA et al., 2015; BARBOSA; SOUZA; FREITAS, 2015; SHAHINA et al. 2015).

Diante do desequilíbrio emocional ocasionado pelo consumo de bebidas alcoólicas, onde se estabelece uma possível crise conjugal, devido ao desentendimento entre o casal, pavimenta-se o constructo da ocorrência de ações e reações no campo físico, psicológico, patrimonial, moral e sexual como demonstram os achados. Ante estas situações de conflito conjugal, o consumo de bebidas alcoólicas catalisa a concepção já existente de uma relação de poder e subserviência dentro da conjugalidade, favorecendo consumação e perenização da violência por parceiro íntimo, pois mantém as desigualdades que são sustentadas pelos estereótipos de gênero com valorização das concepções patriarcais (SENA et al., 2015; BARBOSA; SOUZA; FREITAS, 2015).

\section{Conclusão}

A literatura nacional e internacional desponta para o consumo de bebidas alcoólicas dentro da relação conjugal, como um elemento que contribui de forma significativa para uma evidente propensão à prática da VPI, pois está relacionado com a instabilidade emocional e a reação contra as agressões perpetradas pelo parceiro íntimo.

Diante desse resultado, a criação e a implementação de políticas públicas que trabalhem com a questão de bebidas alcoólicas dentro da relação conjugal é condição sine qua non para o enfrentamento da violência por parceiro íntimo. Deste modo, faz-se necessário o desenvolvimento de campanhas informativas e de intervenções diretas de amplo alcance na coletividade, em todas as faixas etárias, visto que a violência dentro da conjugalidade provoca destruição familiar, visto que não atinge apenas o casal, mas todas as pessoas no entorno dessa relação adoecida, ganhando o efeito bola de neve e atingindo, por sua vez, a sociedade, com reflexos diretos nas relações sociais e consequente elevação de gastos no Sistema Único de Saúde.

A principal limitação do estudo foi a proposta de estudar única e exclusivamente a bebida alcoólica como um dos fatores de risco à ocorrência de violência por parceiro íntimo, bem como a busca em um número limitado de bases de dados. Entende-se, portanto, que é de suma importância também estudar o consumo de bebida alcoólica consorciado com outras drogas e sua influência no engendro da VPI. É necessário, também, que se façam estudos que investiguem a influência do consumo de drogas ilícitas na prática da violência nos espaços da conjugalidade.

\section{Referências}

ANINDITA, D. et al. Local Social Support Mitigates
Depression among Women Contending with Spousal Violence and Husband's Risky Drinking in Mumbai Slum Communities. Journal of Affective Disorders. v. 145, n. 1, p. 126-129, 2013.

BARBOSA, J. A. G.; SOUZA, M. C. M. R. de; FREITAS, M. I. de F.; Violência sexual: narrativas de mulheres com transtornos mentais no Brasil. Pan Americal Journal of Public Health, v. 37, n. 4, p. 273-278, 2015.

BRASIL, Lei 11.340/2006, de 7 de agosto de 2006. Cria mecanismos para coibir a violência doméstica e familiar contra a mulher; dispõe sobre a criação dos Juizados de Violência Doméstica e Familiar contra a Mulher; altera o Código Penal e a Lei de Execução Penal; e dá outras providências. Diário Oficial da União, Brasília, DF, 8 ago. 2006.

Lei 12.853, de 14 de Agosto de 2013. Altera os arts. $5^{\circ}, 68,97,98,99$ e 100, acrescenta arts. 98-A, 98-B, 98-C, 99-A, 99-B, 100-A, 100-B e 109-A e revoga o art. 94 da Lei $\mathrm{n}^{\circ}$ 9.610, de 19 de fevereiro de 1998, para dispor sobre a gestão coletiva de direitos autorais, e dá outras providências. Diário oficial [da] República Federativa do Brasil, Brasília, DF, 14 ago. 2013.

EATON, L. A. et al. Pregnancy, alcohol intake, and intimate partner violence among men and women attending drinking establishments in a Cape Town, South Africa township.

Journal Community Health. v. 37, n. 1, p. 208-216, 2012.

GIMÉNEZ, C. L.; GARCÍA, M. R.; MANCHA, I. H.

Relación entre el consumo abusivo de alcohol y la violencia ejercida por el hombre contra su pareja en la unidad de valoración integral de violencia de género (UVIVG) de Sevilla. Cuad Med Forense, v. 20, n. 4, p. 151-169, 2014.

LINDNER, S. R. et. al. Prevalência de violência física por parceiro íntimo em homens e mulheres de Florianópolis, Santa Catarina, Brasil: estudo de base populacional. Cad. Saúde Pública, Rio de Janeiro, v. 31, n. 4, p. 815-826, 2015.

MENDES, K. D. S.; SILVEIRA, R. C. C. P.; GALVÃO, C. M. Revisão integrativa: método de pesquisa para a incorporação de evidências na saúde e na enfermagem. Texto Contexto Enferm. v. 17, n. 4, p. 758-64, 2008.

OLOWOOKERE, S. A. et al. Patterns and Correlates of Intimate Partner Violence to Women Living With HIV/ AIDS in Osogbo, Southwest Nigeria. Sage Journals. v. 21, n. 11, p. 1330-1340, 2015.

SHAHINA, B. et al. Socio-demographic factors associated with domestic violence in urban slums, Mumbai,

Maharashtra, India. Journal of Medical Research, v. 141, n. 6, p. 783-788, 2015.

SEMAHEGN, A.; BELACHEW, T.; ABDULAHI, M. Domestic violence and its predictors among married women in reproductive age in Fagitalekoma Woreda, Awi 
zone, Amhara regional state, North Western Ethiopia.

Reproductive Health, v.10, p. 63, 2013.

SENA, C. D. et al. Ocorrência de violência doméstica em mulheres que tiveram parto prematuro. Revista Baiana de Enfermagem, v. 26, n. 2, p. 488-497, 2015.

SOUSA, A. R. et al. Repercussions of imprisonment for conjugal violence: discourses of men. Rev. Latino-Am. Enfermagem, v. 24, n. e2847, 2016.

VIEIRA, E. M.; PERDONA, G. S. C.; SANTOS, M. A. Fatores associados à violência Física por parceiro íntimo em usuárias de serviços de saúde. Rev. Saúde Pública, v. 45, n. 4, p. 730-7, 2011.

ZALESKI, M. et al. Violência entre parceiros íntimos e consumo de álcool. Rev Saúde Pública, v. 44, n. 1, p. 5359, 2010.

Recebido em: 01/06/2017

Aceito em:05/02/2018 